\title{
QUANTITATIVE APPROXIMATIONS OF THE LYAPUNOV EXPONENT OF A RATIONAL FUNCTION OVER VALUED FIELDS
}

\author{
YÛSUKE OKUYAMA
}

\begin{abstract}
We establish a quantitative approximation formula of the Lyapunov exponent of a rational function of degree more than one over an algebraically closed field of characteristic 0 that is complete with respect to a non-trivial and possibly non-archimedean absolute value, in terms of the multipliers of periodic points of the rational function. This quantifies both our former convergence result over general fields and the one-dimensional version of Berteloot-Dupont-Molino's one over archimedean fields.
\end{abstract}

\section{INTRODUCTION}

In this article, we establish a quantitative logarithmic equidistribution result for periodic points of a rational function over a more general field than that of the complex numbers, using potential theory on the Berkovich projective line. Let $K$ be an algebraically closed field of characteristic 0 that is complete with respect to a non-trivial and possibly non-archimedean absolute value $|\cdot|$. We note that $K \cong \mathbb{C}$ if and only if $K$ is archimedean. The Berkovich projective line $\mathrm{P}^{1}=\mathrm{P}^{1}(K)$ over $K$ is a compactification of the (classical) projective line $\mathbb{P}^{1}=\mathbb{P}^{1}(K)$ over $K$ and contains $\mathbb{P}^{1}$ as a dense subset. We also note that $\mathrm{P}^{1} \cong \mathbb{P}^{1}$ if and only if $K$ is archimedean.

Let $f \in K(z)$ be a rational function over $K$ of degree $>1$, and let $\mu_{f}$ be the equilibrium (or canonical) measure of $f$ on $\mathrm{P}^{1}$. The chordal derivative $f^{\#}$ of $f$ with respect to the normalized chordal metric on $\mathbb{P}^{1}$ extends to a continuous function on $\mathrm{P}^{1}$. The multiplier of a fixed point $w \in \mathbb{P}^{1}$ of $f^{n}$ for some $n \in \mathbb{N}$ is denoted by $\left(f^{n}\right)^{\prime}(w)$. For every fixed point $w \in \mathbb{P}^{1}$ of $f$, we have $f^{\#}(w)=\left|f^{\prime}(w)\right|$, and the function $\log \left(f^{\#}\right)$ on $\mathrm{P}^{1}$ has a logarithmic singularity at each critical point of $f$ in $\mathbb{P}^{1}$. The Lyapunov exponent of $f$ with respect to $\mu_{f}$ is defined by

$$
L(f):=\int_{\mathrm{P}^{1}} \log \left(f^{\#}\right) \mathrm{d} \mu_{f},
$$

which is in $(-\infty, \infty)$.

Our principal result is the following quantitative approximation of $L(f)$ by the $\log$ of the moduli of the multipliers of non-superattracting periodic points of $f$ in $\mathbb{P}^{1}$, the qualitative version (i.e., with no non-trivial order estimates) of which was obtained in [17, Theorem 1] (see also Szpiro-Tucker

Date: September 17, 2018.

2010 Mathematics Subject Classification. Primary 37P50; Secondary 11S82.

Key words and phrases. periodic point, Lyapunov exponent, quantitative approximation, non-archimedean dynamics, complex dynamics. 
[24] for the qualitative version when $f$ is defined over a number field or a function field): for each $n \in \mathbb{N}$, let $\operatorname{Fix}\left(f^{n}\right)$ be the set of all fixed points of $f^{n}$ in $\mathbb{P}^{1}$, and $\operatorname{Fix}^{*}\left(f^{n}\right)$ be the set of all periodic points $w$ of $f$ in $\mathbb{P}^{1}$ having the exact period $n$ in that $w \in \operatorname{Fix}\left(f^{n}\right) \backslash\left(\bigcup_{m \in \mathbb{N}: m \mid n}\right.$ and $m<n$ Fix $\left.\left(f^{m}\right)\right)$.

Theorem 1. Let $f \in K(z)$ be a rational function of degree $d>1$ over an algebraically closed field $K$ of characteristic 0 that is complete with respect to a non-trivial and possibly non-archimedean absolute value $|\cdot|$. Then

$$
\begin{aligned}
& L(f)=\frac{1}{n d^{n}} \sum_{w \in \operatorname{Fix}\left(f^{n}\right):\left(f^{n}\right)^{\prime}(w) \neq 0} \log \left|\left(f^{n}\right)^{\prime}(w)\right|+O\left(n d^{-n}\right) \quad \text { as } n \rightarrow \infty, \quad \text { and } \\
& \text { (1.3) } \quad L(f)=\frac{1}{n d^{n}} \sum_{w \in \operatorname{Fix}^{*}\left(f^{n}\right)} \log \left|\left(f^{n}\right)^{\prime}(w)\right|+O\left(d^{-n / 2}\right) \quad \text { as } n \rightarrow \infty .
\end{aligned}
$$

In the case that $f$ has at most finitely many attracting periodic points in $\mathbb{P}^{1}$, Theorem 1 yields the following quantitative approximations of $L(f)$ by the multipliers of repelling periodic points of $f$ in $\mathbb{P}^{1}$ : for each $n \in \mathbb{N}$, set $R\left(f^{n}\right):=\left\{w \in \operatorname{Fix}\left(f^{n}\right):\left|\left(f^{n}\right)^{\prime}(w)\right|>1\right\}$ and $R^{*}\left(f^{n}\right):=\left\{w \in \operatorname{Fix}^{*}\left(f^{n}\right):\right.$ $\left.\left|\left(f^{n}\right)^{\prime}(w)\right|>1\right\}$.

Theorem 2. Let $f \in K(z)$ be a rational function of degree $d>1$ over an algebraically closed field $K$ of characteristic 0 that is complete with respect to a non-trivial and possibly non-archimedean absolute value $|\cdot|$.

If $f$ has at most finitely many attracting periodic points in $\mathbb{P}^{1}$, then

$$
\begin{gathered}
L(f)=\frac{1}{n d^{n}} \sum_{w \in R\left(f^{n}\right)} \log \left|\left(f^{n}\right)^{\prime}(w)\right|+O\left(n d^{-n}\right) \quad \text { as } n \rightarrow \infty, \quad \text { and } \\
L(f)=\frac{1}{n d^{n}} \sum_{w \in R^{*}\left(f^{n}\right)} \log \left|\left(f^{n}\right)^{\prime}(w)\right|+O\left(d^{-n / 2}\right) \quad \text { as } n \rightarrow \infty
\end{gathered}
$$

For archimedean $K$, the finiteness assumption in Theorem 2 always holds (cf. [16. Theorem 8.6]); for non-archimedean $K$, any periodic point of the polynomial $f(z)=z^{d}$ is (super) attracting if $|d|<1$. The qualitative version (i.e., with no non-trivial order estimates) of Theorem 2 for archimedean $K$ also follows from Berteloot-Dupont-Molino [7, Corollary 1.6]; see also Berteloot [6]. Their proofs are based on the positivity of $L(f)$, which is the case for archimedean $K$ by Ruelle's inequality; for non-archimedean $K$, the polynomial $f(z)=z^{d}$ has $L(f)=\log |d| \leq 0$. Our proofs of Theorems 1 and 2 are independent of whether $L(f)$ is positive or not.

A bit surprisingly, the proofs of Theorems 1 and 2 are independent of the equidistribution theorem for periodic points of $f$ in $\mathbb{P}^{1}$ towards the equilibrium measure $\mu_{f}$, which was due to Lyubich [15, Theorem 3] for archimedean $K$ and due to Favre-Rivera-Letelier [12, Théorème B] for non-archimedean $K$ of characteristic 0 .

Organization of this article. In Section 2, we prepare a background on dynamics of rational functions over general fields. In Section 3, we show Theorem 1: let us remark that the proof can be simplified if there are no superattracting periodic points (see Lemma 3.1). In Section 4, we show Theorem 2 based on Theorem 1 , 


\section{BACKGROUND}

For the foundations of potential theory on $\mathrm{P}^{1}$, see [4, $\S 5$ and $\left.\S 8\right]$, [11, $\left.\S 7\right]$, [14, §1-§4], [26, Chapter III]. For a potential theoretic study of dynamics on $\mathrm{P}^{1}$, see [4, §10], [12, §3], [14, §5], [8, Chapitre VIII]. See also [5], [21] including non-archimedean dynamics.

Chordal metric on $\mathbb{P}^{1}$. Let $K$ be an algebraically closed field complete with respect to a non-trivial and possibly non-archimedean absolute value $|\cdot|$. For a while, we allow $K$ to have any characteristic. Let $\left\|\left(p_{0}, p_{1}\right)\right\|$ be the maximum norm $\max \left\{\left|p_{0}\right|,\left|p_{1}\right|\right\}$ on $K^{2}$ (for non-archimedean $K$ ) or the Euclidean norm $\sqrt{\left|p_{0}\right|^{2}+\left|p_{1}\right|^{2}}$ on $K^{2}$ (for archimedean $K$ ). The origin of $K^{2}$ is also denoted by 0 , and $\pi$ is the canonical projection $K^{2} \backslash\{0\} \rightarrow \mathbb{P}^{1}=$ $\mathbb{P}^{1}(K)$. Setting the wedge product $\left(z_{0}, z_{1}\right) \wedge\left(w_{0}, w_{1}\right):=z_{0} w_{1}-z_{1} w_{0}$ on $K^{2} \times K^{2}$, the normalized chordal metric $[z, w]$ on $\mathbb{P}^{1}$ is the function

$$
(z, w) \mapsto[z, w]:=|p \wedge q| /(\|p\| \cdot\|q\|)(\leq 1)
$$

on $\mathbb{P}^{1} \times \mathbb{P}^{1}$, where $p \in \pi^{-1}(z), q \in \pi^{-1}(w)$. Although the topology of the Berkovich projective line $\mathrm{P}^{1}=\mathrm{P}^{1}(K)$, which is a compactification of $\mathbb{P}^{1}$, is not always metrizable, the relative topology of $\mathbb{P}^{1}$ coincides with the metric topology on $\mathbb{P}^{1}$ induced by the normalized chordal metric.

Hsia kernel on $\mathrm{P}^{1}$. Let $\delta_{\mathcal{S}}$ be the Dirac measure on $\mathrm{P}^{1}=\mathrm{P}^{1}(K)$ at $\mathcal{S} \in$ $\mathrm{P}^{1}$. Let $\Omega_{\text {can }}$ be the Dirac measure $\delta_{\mathcal{S}_{\text {can }}}$ at the canonical (or Gauss) point $\mathcal{S}_{\text {can }} \in \mathrm{P}^{1}$ for non-archimedean $K([4, \S 1.2],[12, \S 2.1])$ or the Fubini-Study area element $\omega$ on $\mathbb{P}^{1}$ normalized as $\omega\left(\mathbb{P}^{1}\right)=1$ for archimedean $K$. For nonarchimedean $K$, the normalized chordal metric on $\mathbb{P}^{1}$ canonically extends to the generalized Hsia kernel $\left[\mathcal{S}, \mathcal{S}^{\prime}\right]_{\text {can }}$ with respect to $\mathcal{S}_{\text {can }}$ on $\mathrm{P}^{1}$ (for the construction, see [4, §4.4], [12, §2.1]), which vanishes if and only if $\mathcal{S}=\mathcal{S}^{\prime} \in$ $\mathbb{P}^{1}$. For archimedean $K,[z, w]_{\text {can }}$ is defined by $[z, w]$, by convention. Let $\Delta$ be the Laplacian on $\mathrm{P}^{1}$ (for the construction in the non-archimedean case, see $[4, \S 5],[11, \S 7.7],[25, \S 3])$ normalized so that for each $\mathcal{S} \in \mathrm{P}^{1}$,

$$
\Delta \log [\cdot, \mathcal{S}]_{\text {can }}=\delta_{\mathcal{S}}-\Omega_{\text {can }} \quad \text { on } \mathrm{P}^{1}
$$

(for non-archimedean $K$, see [4, Example 5.19], [12, §2.4]: in [4 the opposite sign convention on $\Delta$ is adopted).

Potential theory on $\mathrm{P}^{1}$. A continuous weight $g$ on $\mathrm{P}^{1}$ is a continuous function on $\mathrm{P}^{1}$ such that $\mu^{g}:=\Delta g+\Omega_{\text {can }}$ is a probability Radon measure on $\mathrm{P}^{1}$. For a continuous weight $g$ on $\mathrm{P}^{1}$, the $g$-potential kernel

$$
\Phi_{g}\left(\mathcal{S}, \mathcal{S}^{\prime}\right):=\log \left[\mathcal{S}, \mathcal{S}^{\prime}\right]_{\text {can }}-g(\mathcal{S})-g\left(\mathcal{S}^{\prime}\right)
$$

(the negative of an Arakelov Green function of $\mu^{g}$ ) on $\mathrm{P}^{1}$ is an upper semicontinuous function on $\mathrm{P}^{1} \times \mathrm{P}^{1}$. The (exp of the) $\Phi_{g}$ is separately continuous in each of the variables $\mathcal{S}, \mathcal{S}^{\prime} \in \mathrm{P}^{1}$, and it introduces the $g$-potential

$$
U_{g, \nu}(\cdot):=\int_{\mathrm{P}^{1}} \Phi_{g}(\cdot, \mathcal{S}) \mathrm{d} \nu(\mathcal{S})
$$

on $\mathrm{P}^{1}$ of each Radon measure $\nu$ on $\mathrm{P}^{1}$. By the Fubini theorem, $\Delta U_{g, \nu}=$ $\nu-\nu\left(\mathrm{P}^{1}\right) \mu^{g}$ on $\mathrm{P}^{1}$. The $g$-equilibrium energy $V_{g} \in[-\infty,+\infty)$ of $\mathrm{P}^{1}$ is the 
supremum of the energy functional

$$
\nu \mapsto \int_{\mathrm{P}^{1} \times \mathrm{P}^{1}} \Phi_{g} \mathrm{~d}(\nu \times \nu)
$$

on the space of all probability Radon measures on $\mathrm{P}^{1}$. Indeed $V_{g} \in(-\infty, \infty)$ since $\int_{\mathrm{P}^{1} \times \mathrm{P}^{1}} \Phi_{g} \mathrm{~d}\left(\Omega_{\text {can }} \times \Omega_{\text {can }}\right)>-\infty$. The variational characterization of $\mu^{g}$ asserts that the above energy functional attains the supremum uniquely at $\nu=\mu^{g}$. Moreover,

$$
U_{g, \mu^{g}} \equiv V_{g} \quad \text { on } \mathrm{P}^{1}
$$

(for non-archimedean $K$, see [4, Theorem 8.67 and Proposition 8.70]). A continuous weight $g$ on $\mathrm{P}^{1}$ is a normalized weight on $\mathrm{P}^{1}$ if $V_{g}=0$. For a continuous weight $g$ on $\mathrm{P}^{1}, \bar{g}:=g+V_{g} / 2$ is the unique normalized weight on $\mathrm{P}^{1}$ satisfying $\mu^{\bar{g}}=\mu^{g}$.

Equilibrium measure $\mu_{f}$. A rational function $f \in K(z)$ of degree $d>1$ extends to a continuous, surjective, open, and discrete endomorphism of $\mathrm{P}^{1}$, preserving $\mathbb{P}^{1}$ and $\mathrm{P}^{1} \backslash \mathbb{P}^{1}$, respectively, and induces a push-forward $f_{*}$ and a pullback $f^{*}$ on the space of continuous functions on $\mathrm{P}^{1}$ and, by duality, on the space of Radon measures on $\mathrm{P}^{1}([4, \S 9]$, [12, §2.2]). A non-degenerate homogeneous lift $F=\left(F_{0}, F_{1}\right)$ of (the unextended) $f$ is a homogeneous polynomial endomorphism on $K^{2}$ such that $\pi \circ F=f \circ \pi$ on $K^{2} \backslash\{0\}$ and that $F^{-1}(0)=\{0\}$. The latter condition is equivalent to $\operatorname{Res} F \in K \backslash\{0\}$ (for the definition of the homogeneous resultant $\operatorname{Res} F=\operatorname{Res}\left(F_{0}, F_{1}\right)$ of $F$, see, e.g., [22, §2.4]). Such $F$ is unique up to multiplication in $K \backslash\{0\}$, and has the algebraic degree $d$, i.e., $\operatorname{deg} F_{0}=\operatorname{deg} F_{1}=\operatorname{deg} f=d$. For every $n \in \mathbb{N}, F^{n}$ is a non-degenerate homogeneous lift of $f^{n}$, and the function

$$
T_{F^{n}}:=\log \left\|F^{n}\right\|-d^{n} \log \|\cdot\|
$$

on $K^{2} \backslash\{0\}$ descends to $\mathbb{P}^{1}$ and in turn extends continuously to $\mathrm{P}^{1}$, satisfying $\Delta T_{F^{n}}=\left(f^{n}\right)^{*} \Omega_{\text {can }}-d^{n} \Omega_{\text {can }}$ on $\mathrm{P}^{1}$ (see, e.g., [18, Definition 2.8]). The uniform limit $g_{F}:=\lim _{n \rightarrow \infty} T_{F^{n}} / d^{n}$ on $\mathrm{P}^{1}$ exists and is indeed a continuous weight on $\mathrm{P}^{1}$. The equilibrium (or canonical) measure of $f$ is the probability Radon measure

$$
\mu_{f}:=\Delta g_{F}+\Omega_{\text {can }}=\lim _{n \rightarrow \infty} d^{-n}\left(f^{n}\right)^{*} \Omega_{\text {can }} \quad \text { weakly on } \mathrm{P}^{1},
$$

which is independent of the choice of the lift $F$ and satisfies that $f^{*} \mu_{f}=$ $d \cdot \mu_{f}$ and $f_{*} \mu_{f}=\mu_{f}$ on $\mathrm{P}^{1}$ (for non-archimedean $K$, see [4, §10], [9, §2], [12, §3.1]). The dynamical Green function $g_{f}$ of $f$ on $\mathrm{P}^{1}$ is the unique normalized weight on $\mathrm{P}^{1}$ such that $\mu^{g_{f}}=\mu_{f}$. By the energy formula $V_{g_{F}}=$ $-(\log |\operatorname{Res} F|) /(d(d-1))$ (due to DeMarco [10] for archimedean $K$ and due to Baker-Rumely [3] when $f$ is defined over a number field; see 2, Appendix A] for a simple proof which works for general $K$ ) and $\operatorname{Res}(c F)=c^{2 d} \operatorname{Res} F$ for each $c \in K \backslash\{0\}$ (cf. [22, Proposition 2.13(b)]), there is a non-degenerate homogeneous lift $F$ of $f$ satisfying $V_{g_{F}}=0$, or equivalently, that $g_{F}=g_{f}$ on $\mathrm{P}^{1}$. We note that $U_{g_{f}, \mu_{f}} \equiv 0$ on $\mathrm{P}^{1}$ and that for every $n \in \mathbb{N}, \mu_{f^{n}}=\mu_{f}$ and $g_{f^{n}}=g_{f}$ on $\mathrm{P}^{1}$. 
Logarithmic proximity function $\Phi\left(f^{n}, \mathrm{Id}\right)_{g_{f}}$. For more details on the following, see [18, Proposition 2.9].

Proposition 2.1. For rational functions $\phi_{i} \in K(z)$ of degree $d_{i}, i \in\{1,2\}$, on $\mathbb{P}^{1}$ satisfying $\phi_{1} \not \equiv \phi_{2}$ and $\max \left\{d_{1}, d_{2}\right\}>0$, the function $z \mapsto\left[\phi_{1}(z), \phi_{2}(z)\right]$ on $\mathbb{P}^{1}$ extends continuously to a function $\mathcal{S} \mapsto\left[\phi_{1}, \phi_{2}\right]_{\text {can }}(\mathcal{S})$ on $\mathrm{P}^{1}$.

For each $n \in \mathbb{N}$, we introduce the logarithmic proximity function weighted by $g_{f}$

$$
\Phi\left(f^{n}, \mathrm{Id}\right)_{g_{f}}(\cdot):=\log \left[f^{n}, \mathrm{Id}\right]_{\operatorname{can}}(\cdot)-g_{f} \circ f^{n}(\cdot)-g_{f}(\cdot)
$$

between $f^{n}$ and $\mathrm{Id}$ on $\mathrm{P}^{1}$, and set

$$
\left[f^{n}=\mathrm{Id}\right]:=\sum_{w \in \mathbb{P}^{1}: f^{n}(w)=w} \delta_{w} \quad \text { on } \mathrm{P}^{1},
$$

where the sum takes into account the multiplicity of each root $w \in \mathbb{P}^{1}$ of $f^{n}=\mathrm{Id}$.

For a proof of the following, see, e.g., [18, Lemma 2.19].

Lemma 2.2 (cf. [23, (1.4)]). For every $n \in \mathbb{N}$,

$$
\Phi\left(f^{n}, \mathrm{Id}\right)_{g_{f}}(\cdot)=U_{g_{f},\left[f^{n}=\mathrm{Id}\right]-\left(d^{n}+1\right) \mu_{f}}+\int_{\mathrm{P}^{1}} \Phi\left(f^{n}, \mathrm{Id}\right)_{g_{f}} \mathrm{~d} \mu_{f}
$$

on $\mathrm{P}^{1}$. Since $U_{g_{f}, \mu_{f}} \equiv 0$ on $\mathrm{P}^{1}$, this is rewritten as

$$
\Phi\left(f^{n}, \mathrm{Id}\right)_{g_{f}}(\cdot)=U_{g_{f},\left[f^{n}=\mathrm{Id}\right]}+\int_{\mathrm{P}^{1}} \Phi\left(f^{n}, \mathrm{Id}\right)_{g_{f}} \mathrm{~d} \mu_{f} \quad \text { on } \mathrm{P}^{1} .
$$

Chordal derivative $f^{\#}$. The multiplier of a fixed point $w \in \mathbb{P}^{1}$ of $f^{n}$ for some $n \in \mathbb{N}$ is denoted by $\left(f^{n}\right)^{\prime}(w)$. A fixed point $w \in \mathbb{P}^{1}$ of $f^{n}$ for some $n \in \mathbb{N}$ is said to be superattracting, attracting, or repelling if $\left(f^{n}\right)^{\prime}(w)=0$, $\left|\left(f^{n}\right)^{\prime}(w)\right|<1$, or $\left|\left(f^{n}\right)^{\prime}(w)\right|>1$, respectively.

In the rest of this subsection, we suppose that $K$ has characteristic 0 .

Notation 2.3. Let $C(f)$ be the set of all critical points $c$ of $f$ in $\mathbb{P}^{1}$, i.e., $f^{\prime}(c)=0$.

For every $n \in \mathbb{N}, f^{n}$ has $2 d^{n}-2$ critical points in $\mathbb{P}^{1}$ if we take into account the multiplicity of each $c \in C\left(f^{n}\right)$. Let $S A T(f)$ be the set of all superattracting periodic points of $f$ in $\mathbb{P}^{1}$, i.e.,

$$
S A T(f)=\bigcup_{n \in \mathbb{N}}\left(\operatorname{Fix}\left(f^{n}\right) \cap C\left(f^{n}\right)\right) .
$$

By $\# C(f)<\infty$ and the chain rule, $\# S A T(f)<\infty$.

The chordal derivative $f^{\#}$ on $\mathbb{P}^{1}$ is a function

$$
\mathbb{P}^{1} \ni z \mapsto f^{\#}(z):=\lim _{\mathbb{P}^{1} \ni w \rightarrow z}[f(w), f(z)] /[w, z] .
$$

For every non-degenerate homogeneous lift $F$ of $f$, there exists a sequence $\left(C_{j}^{F}\right)_{j=1}^{2 d-2}$ in $K^{2} \backslash\{0\}$ such that the Jacobian determinant of $F$ factors as

$$
\operatorname{det} D F(\cdot)=\prod_{j=1}^{2 d-2}\left(\cdot \wedge C_{j}^{F}\right) \quad \text { on } K^{2} .
$$


Setting $c_{j}:=\pi\left(C_{j}^{F}\right)(j=1,2, \ldots, 2 d-2)$, the sequence $\left(c_{j}\right)_{j=1}^{2 d-2}$ in $\mathbb{P}^{1}$ is independent of the choice of the lift $F$ upto permutation, and satisfies that for every $c \in C(f), \#\left\{j \in\{1,2, \ldots, 2 d-2\}: c_{j}=c\right\}-1$ equals the multiplicity of $c$. For every $z \in \mathbb{P}^{1}$, by a computation involving Euler's identity, we have

$$
f^{\#}(z)=\frac{1}{|d|} \frac{\|p\|^{2}}{\|F(p)\|^{2}}|\operatorname{det} D F(p)| \quad \text { for } p \in \pi^{-1}(z)
$$

(cf. 13, Theorem 4.3]), which with (2.1) yields the equality $\log \left(f^{\#}\right)=$ $-\log |d|+\sum_{j=1}^{2 d-2}\left(\log \left[\cdot, c_{j}\right]+\log \left\|C_{j}^{F}\right\|\right)-2 T_{F} \mid \mathbb{P}^{1}$ on $\mathbb{P}^{1}$. The (exp of the) right hand side extends $f^{\#}$ to a continuous function on $\mathrm{P}^{1}$ so that

$$
\log \left(f^{\#}\right)=-\log |d|+\sum_{j=1}^{2 d-2}\left(\log \left[\cdot, c_{j}\right]_{\mathrm{can}}+\log \left\|C_{j}^{F}\right\|\right)-2 T_{F} \quad \text { on } \mathrm{P}^{1},
$$

where the continuous extension of $f^{\#}$ is also denoted by the same $f^{\#}$. The chain rule for $f^{\#}$ on $\mathbb{P}^{1}$ extends to $\mathrm{P}^{1}$.

For completeness, we include a proof of the following.

Lemma 2.4 ([19, Lemma 3.6]). On $\mathrm{P}^{1}$,

$$
\log \left(f^{\#}\right)=L(f)+\sum_{c \in C(f)} \Phi_{g_{f}}(\cdot, c)+2 g_{f} \circ f-2 g_{f} .
$$

Here the sum over $C(f)$ takes into account the multiplicity of each $c \in C(f)$.

Proof. Let us choose a non-degenerate homogeneous lift $F$ of $f$ so that $g_{F}=g_{f}$, i.e., $g_{f}=\lim _{n \rightarrow \infty} T_{F^{n}} / d^{n}$ on $\mathrm{P}^{1}$.

By the definition of $\Phi_{g_{f}}$, (2.5) is rewritten as

$\log \left(f^{\#}\right)=-\log |d|+\sum_{j=1}^{2 d-2}\left(\Phi_{g_{f}}\left(\cdot, c_{j}\right)+g_{f}\left(c_{j}\right)+\log \left\|C_{j}^{F}\right\|\right)-2 T_{F}+(2 d-2) g_{f}$

on $\mathrm{P}^{1}$. We claim that $g_{f} \circ f=d \cdot g_{f}-T_{F}$ on $\mathrm{P}^{1}$, which is equivalent to $-2 T_{F}+(2 d-2) g_{f}=2 g_{f} \circ f-2 g_{f}$ on $\mathrm{P}^{1}$; Indeed, for every $z \in \mathbb{P}^{1}$, by $g_{f}=g_{F}=\lim _{n \rightarrow \infty} T_{F^{n}} / d^{n}$ on $\mathrm{P}^{1}$, we have

$$
\begin{array}{r}
g_{f} \circ f(z)-d \cdot g_{f}(z)=\lim _{n \rightarrow \infty}\left(\frac{1}{d^{n}}\left(\log \left\|F^{n}(F(p))\right\|-d^{n} \log \|F(p)\|\right)\right) \\
-d \cdot \lim _{n \rightarrow \infty}\left(\frac{1}{d^{n+1}}\left(\log \left\|F^{n+1}(p)\right\|-d^{n+1} \log \|p\|\right)\right) \\
=-(\log \|F(p)\|-d \cdot \log \|p\|)=-T_{F}(z),
\end{array}
$$

where $p \in \pi^{-1}(z)$. Hence $g_{f} \circ f-d \cdot g_{f}=-T_{F}$ on $\mathbb{P}^{1}$, which in turn holds on $\mathrm{P}^{1}$ by the continuity of both sides, and the claim holds.

By this claim, (2.5I) is rewritten as

$$
\log \left(f^{\#}\right)=-\log |d|+\sum_{j=1}^{2 d-2}\left(\Phi_{g_{f}}\left(\cdot, c_{j}\right)+g_{f}\left(c_{j}\right)+\log \left\|C_{j}^{F}\right\|\right)+2 g_{f} \circ f-2 g_{f} \quad \text { on } \mathrm{P}^{1} .
$$


Integrating both sides in (2.7) against $\mathrm{d} \mu_{f}$ over $\mathrm{P}^{1}$, by $U_{g_{f}, \mu_{f}} \equiv 0$ and $f_{*} \mu_{f}=\mu_{f}$ on $\mathrm{P}^{1}$, we have

$$
\begin{aligned}
& L(f):=\int_{\mathrm{P}^{1}} \log \left(f^{\#}\right) \mathrm{d} \mu_{f} \\
= & -\log |d|+\sum_{j=1}^{2 d-2}\left(U_{g_{f}, \mu_{f}}\left(c_{j}\right)+g_{f}\left(c_{j}\right)+\log \left\|C_{j}^{F}\right\|\right)+2 \int_{\mathrm{P}^{1}} g_{f} \circ f \mathrm{~d} \mu_{f}-2 \int_{\mathrm{P}^{1}} g_{f} \mathrm{~d} \mu_{f} \\
= & -\log |d|+\sum_{j=1}^{2 d-2}\left(g_{f}\left(c_{j}\right)+\log \left\|C_{j}^{F}\right\|\right) .
\end{aligned}
$$

This with (2.7) completes the proof of Lemma 2.4,

Berkovich Julia and Fatou sets $\mathrm{J}(f)$ and $\mathrm{F}(f)$. The exceptional set of (the extended) $f$ is $E(f):=\left\{a \in \mathbb{P}^{1}: \# \bigcup_{n \in \mathbb{N}} f^{-n}(a)<\infty\right\}$, which agrees with the set of all $a \in S A T(f)$ such that $\operatorname{deg}_{f^{j}(a)} f=d$ for any $j \in \mathbb{N}$. The Berkovich Julia set of $f$ is

$$
\mathrm{J}(f):=\left\{\mathcal{S} \in \mathrm{P}^{1}: \underset{V: \text { open in } \mathrm{P}^{1} \text { and contains } \mathcal{S}}{ }\left(\bigcup_{n \in \mathbb{N}} f^{n}(V)\right)=\mathrm{P}^{1} \backslash E(f)\right\}
$$

(cf. [12, Definition 2.8]), which is closed in $\mathrm{P}^{1}$, and the Berkovich Fatou set of $f$ is $\mathrm{F}(f):=\mathrm{P}^{1} \backslash \mathrm{J}(f)$, which is open in $\mathrm{P}^{1}$. For archimedean $K$, these definitions of $\mathrm{J}(f)$ and $\mathrm{F}(f)$ are equivalent to those of the Julia and Fatou sets of $f$ in terms of the non-normality and the normality of $\left\{f^{n}: n \in \mathbb{N}\right\}$, respectively.

A Berkovich Fatou component of $f$ is a connected component of $\mathrm{F}(f)$; if $W$ is a Berkovich Fatou component of $f$, then so is $f(W)$. A Berkovich Fatou component $W$ of $f$ is cyclic under $f$ if $f^{p}(W)=W$ for some $p \in \mathbb{N}$. For archimedean $K$, the classification of cyclic (Berkovich) Fatou components into

- immediate attractive basins of either (super)attracting or parabolic cycles, and

- rotation domains, i.e., Siegel disks and Herman rings

is essentially due to Fatou (cf. [16, Theorem 5.2]). For non-archimedean $K$, its counterpart due to Rivera-Letelier (see [12, Proposition 2.16] and its esquisse de démonstration and also [5, Remark 7.10]) asserts that every cyclic Berkovich Fatou component $W$ of $f$ is either

- an immediate attractive basin of $f$ in that $W$ contains a (super) attracting fixed point a of $f^{p}$ in $W \cap \mathbb{P}^{1}$ for some $p \in \mathbb{N}$ and that $\lim _{n \rightarrow \infty}\left(f^{p}\right)^{n}(w)=$ a for any $w \in W$, or

- a singular domain of $f$ in that $f^{p}(W)=W$ and that $f^{p}: W \rightarrow W$ is injective for some $p \in \mathbb{N}$,

and moreover, only one of these two possibilities occurs. 


\section{Proof of Theorem 1}

Let $K$ be an algebraically closed field of characteristic 0 that is complete with respect to a non-trivial and possibly non-archimedean absolute value $|\cdot|$. Let $f \in K(z)$ be a rational function over $K$ of degree $d>1$.

We note that $\left[f^{n}=\mathrm{Id}\right] /\left(d^{n}+1\right)$ is a probability Radon measure on $\mathrm{P}^{1}$, and that $\sup _{n \in \mathbb{N}}\left(\left[f^{n}=\mathrm{Id}\right](S A T(f))\right) \leq \# S A T(f)<\infty$ for every $n \in \mathbb{N}$.

The strategy of the proof of Theorem 1 is to compute the difference $\int_{\mathrm{P}^{1} \backslash S A T(f)} \log \left(f^{\#}\right) \mathrm{d}\left[f^{n}=\mathrm{Id}\right]-\left(\left[f^{n}=\mathrm{Id}\right]\left(\mathrm{P}^{1} \backslash S A T(f)\right)\right) L(f)$ in two different ways, by integrating the equality (2.6) applied to $f^{n}$ and the (2.6) itself against $\left[f^{n}=\mathrm{Id}\right]$ over $\mathrm{P}^{1} \backslash S A T(f)$, for each $n \in \mathbb{N}$.

Lemma 3.1. For every $n \in \mathbb{N}$,

$$
\begin{aligned}
& \frac{1}{d^{n}} \int_{\mathrm{P}^{1} \backslash S A T(f)} \log \left(f^{\#}\right) \mathrm{d}\left[f^{n}=\mathrm{Id}\right]-\frac{\left[f^{n}=\mathrm{Id}\right]\left(\mathrm{P}^{1} \backslash S A T(f)\right)}{d^{n}} L(f) \\
= & \frac{1}{n d^{n}} \sum_{c \in\left(C\left(f^{n}\right) \backslash \operatorname{Fix}\left(f^{n}\right)\right) \cap\left(\bigcup_{j=0}^{n-1} f^{-j}(C(f) \backslash S A T(f))\right)} \Phi_{g_{f}}\left(f^{n}(c), c\right) \\
& +\frac{1}{n d^{n}} \sum_{c \in\left(C\left(f^{n}\right) \backslash \operatorname{Fix}\left(f^{n}\right)\right) \cap\left(\bigcup_{j=0}^{n-1} f^{-j}(C(f) \cap S A T(f))\right)} \Phi_{g_{f}}\left(f^{n}(c), c\right) \\
& -\frac{1}{n d^{n}} \sum_{c \in C\left(f^{n}\right) \backslash \operatorname{Fix}\left(f^{n}\right)} \int_{S A T(f)} \Phi_{g_{f}}(c, \cdot) \mathrm{d}\left[f^{n}=\mathrm{Id}\right](\cdot) \\
& -\frac{1}{n d^{n}} \sum_{c \in C\left(f^{n}\right) \cap \operatorname{Fix}\left(f^{n}\right)} \int_{S A T(f) \backslash\{c\}} \Phi_{g_{f}}(c, \cdot) \mathrm{d}\left[f^{n}=\mathrm{Id}\right](\cdot) \\
& -\frac{2-2 d^{-n}}{n} \int_{\mathrm{P}^{1}} \Phi\left(f^{n}, \mathrm{Id}\right)_{g_{f}} \mathrm{~d} \mu_{f} .
\end{aligned}
$$

Here the sums over subsets in $C\left(f^{n}\right)$ take into account the multiplicity of each $c$ as a critical point of $f^{n}$.

Proof. Integrating both sides in (2.6) against $\mathrm{d}[f=\mathrm{Id}]$ over $\mathbb{P}^{1} \backslash S A T(f)$, since $f_{*}[f=\mathrm{Id}]=[f=\mathrm{Id}]$ on $\mathrm{P}^{1} \backslash S A T(f)$, we have

$$
\begin{aligned}
\int_{\mathrm{P}^{1} \backslash S A T(f)} \log (f)^{\#} \mathrm{~d}[f=\mathrm{Id}]-([f & \left.=\mathrm{Id}]\left(\mathrm{P}^{1} \backslash S A T(f)\right)\right) \cdot L(f) \\
& =\sum_{c \in C(f)} U_{g_{f},[f=\mathrm{Id}] \mid\left(\mathrm{P}^{1} \backslash S A T(f)\right)}(c) .
\end{aligned}
$$

We claim that for every $c \in C(f)$,

$$
\begin{aligned}
\left.U_{g_{f},[f}=\mathrm{Id}\right] \mid\left(\mathrm{P}^{1} \backslash S A T(f)\right) & (c)+\int_{\mathrm{P}^{1}} \Phi(f, \mathrm{Id})_{g_{f}} \mathrm{~d} \mu_{f} \\
& =\left\{\begin{array}{cl}
\Phi_{g_{f}}(f(c), c)-\int_{S A T(f)} \Phi_{g_{f}}(c, \cdot) \mathrm{d}[f=\mathrm{Id}](\cdot) & \text { if } c \notin \operatorname{Fix}(f), \\
-\int_{S A T(f) \backslash\{c\}} \Phi_{g_{f}}(c, \cdot) \mathrm{d}[f=\mathrm{Id}](\cdot) & \text { if } c \in \operatorname{Fix}(f) ;
\end{array}\right.
\end{aligned}
$$


Indeed, using (2.4) and $\Phi(f, \mathrm{Id})_{g_{f}}=\Phi_{g_{f}}(f$, Id $)$ on $\mathbb{P}^{1}$, we have

$$
\begin{aligned}
& U_{g_{f},[f=\mathrm{Id}] \mid\left(\mathrm{P}^{1} \backslash S A T(f)\right)}(c)=\lim _{\mathbb{P}^{1} \ni z \rightarrow c} U_{g_{f},[f=\mathrm{Id}] \mid\left(\mathrm{P}^{1} \backslash S A T(f)\right)}(z) \\
= & \lim _{\mathbb{P}^{1} \ni z \rightarrow c}\left(\Phi_{g_{f}}(f(z), z)-\int_{S A T(f)} \Phi_{g_{f}}(z, \cdot) \mathrm{d}[f=\mathrm{Id}](\cdot)\right)-\int_{\mathrm{P}^{1}} \Phi(f, \mathrm{Id})_{g_{f}} \mathrm{~d} \mu_{f},
\end{aligned}
$$

and moreover,

$$
\begin{aligned}
& \lim _{\mathbb{P}^{1} \ni z \rightarrow c}\left(\Phi_{g_{f}}(f(z), z)-\int_{S A T(f)} \Phi_{g_{f}}(z, \cdot) \mathrm{d}[f=\mathrm{Id}](\cdot)\right) \\
& =\left\{\begin{array}{cc}
\Phi_{g_{f}}(f(c), c)-\int_{S A T(f)} \Phi_{g_{f}}(c, \cdot) \mathrm{d}[f=\mathrm{Id}](\cdot) & \text { if } c \notin \operatorname{Fix}(f), \\
\lim _{\mathbb{P}^{1} \ni z \rightarrow c}\left(\Phi_{g_{f}}(f(z), z)-\Phi_{g_{f}}(z, c)\right) & \\
\quad-\int_{S A T(f) \backslash\{c\}} \Phi_{g_{f}}(c, \cdot) \mathrm{d}[f=\mathrm{Id}](\cdot) & \text { if } c \in \operatorname{Fix}(f) .
\end{array}\right.
\end{aligned}
$$

In the latter case that $c \in C(f) \cap \operatorname{Fix}(f)$, the first term in the right hand side is computed as

$$
\begin{aligned}
& \lim _{\mathbb{P}^{1} \ni z \rightarrow c}\left(\Phi_{g_{f}}(f(z), z)-\Phi_{g_{f}}(z, c)\right)=\lim _{\mathbb{P}^{1} \ni z \rightarrow c} \log \frac{[f(z), z]}{[z, c]} \\
& \quad=\lim _{\mathbb{P}^{1} \ni z \rightarrow c} \log \frac{|f(z)-f(c)+c-z|}{|z-c|}=\log \left|f^{\prime}(c)-1\right|=\log |0-1|=0,
\end{aligned}
$$

where we can assume $c \neq \infty$ by the coordinate change $w \mapsto 1 / w$ when $c=\infty$. Hence the claim holds.

For every $n \in \mathbb{N}$, from (3.5) and (3.6) applied to $f^{n}$, we have

$$
\begin{aligned}
& \int_{\mathrm{P}^{1} \backslash S A T(f)} \log \left(f^{n}\right)^{\#} \mathrm{~d}\left[f^{n}=\mathrm{Id}\right]-\left(\left[f^{n}=\mathrm{Id}\right]\left(\mathrm{P}^{1} \backslash S A T(f)\right)\right) \cdot L\left(f^{n}\right) \\
= & \sum_{c \in C\left(f^{n}\right) \backslash \operatorname{Fix}\left(f^{n}\right)} \Phi_{g_{f}}\left(f^{n}(c), c\right)-\sum_{c \in C\left(f^{n}\right) \backslash \operatorname{Fix}\left(f^{n}\right)} \int_{S A T(f)} \Phi_{g_{f}}(c, \cdot) \mathrm{d}\left[f^{n}=\mathrm{Id}\right](\cdot) \\
- & \sum_{c \in C\left(f^{n}\right) \cap \operatorname{Fix}\left(f^{n}\right)} \int_{S A T(f) \backslash\{c\}} \Phi_{g_{f}}(c, \cdot) \mathrm{d}\left[f^{n}=\mathrm{Id}\right](\cdot)-\left(2 d^{n}-2\right) \int_{\mathrm{P}^{1}} \Phi\left(f^{n}, \mathrm{Id}\right)_{g_{f}} \mathrm{~d} \mu_{f},
\end{aligned}
$$

where $g_{f^{n}}=g_{f}, \mu_{f^{n}}=\mu_{f}$, and $S A T\left(f^{n}\right)=S A T(f) \cap \operatorname{Fix}\left(f^{n}\right)$, and by the chain rule (and $f_{*}\left[f^{n}=\mathrm{Id}\right]=\left[f^{n}=\mathrm{Id}\right]$ on $\mathrm{P}^{1} \backslash S A T(f)$ and $f_{*} \mu_{f}=\mu_{f}$ on $\mathrm{P}^{1}$, recalling also the definition (1.1) of $\left.L(f)\right)$, we have

$$
\begin{aligned}
& \int_{\mathrm{P}^{1} \backslash S A T(f)} \log \left(f^{n}\right)^{\#} \mathrm{~d}\left[f^{n}=\mathrm{Id}\right]-\left(\left[f^{n}=\mathrm{Id}\right]\left(\mathrm{P}^{1} \backslash S A T(f)\right)\right) \cdot L\left(f^{n}\right) \\
= & n\left(\int_{\mathrm{P}^{1} \backslash S A T(f)} \log \left(f^{\#}\right) \mathrm{d}\left[f^{n}=\mathrm{Id}\right]-\left(\left[f^{n}=\mathrm{Id}\right]\left(\mathrm{P}^{1} \backslash S A T(f)\right)\right) \cdot L(f)\right) .
\end{aligned}
$$

Now the proof of Lemma 3.1 is complete since $C\left(f^{n}\right)=\bigcup_{j=0}^{n-1} f^{-j}(C(f))$ and $C(f)=(C(f) \backslash S A T(f)) \cup(C(f) \cap S A T(f))$.

Let us estimate the terms (3.1), (3.2), (3.3), and (3.4). 
Lemma 3.2.

$\frac{1}{n d^{n}} \sum_{c \in\left(C\left(f^{n}\right) \backslash \operatorname{Fix}\left(f^{n}\right)\right) \cap\left(\bigcup_{j=0}^{n-1} f^{-j}(C(f) \backslash S A T(f))\right)} \Phi_{g_{f}}\left(f^{n}(c), c\right)=O(1) \quad$ as $n \rightarrow \infty$.

Here, for every $n \in \mathbb{N}$, the sum takes into account the multiplicity of each $c$ as a critical point of $f^{n}$.

Proof. For every $n \in \mathbb{N}$, by the definition of $\Phi_{g_{f}}$, we have

$$
\begin{aligned}
& \frac{2 d^{n}-2}{n d^{n}} \cdot 2 \sup _{\mathrm{P}^{1}}\left|g_{f}\right| \\
\geq & \frac{1}{n d^{n}} \sum_{c \in\left(C\left(f^{n}\right) \backslash \operatorname{Fix}\left(f^{n}\right)\right) \cap\left(\bigcup_{j=0}^{n-1} f^{-j}(C(f) \backslash S A T(f))\right)} \Phi_{g_{f}}\left(f^{n}(c), c\right) \\
\geq & \frac{1}{n d^{n}} \sum_{c^{\prime} \in C(f) \backslash S A T(f)} \sum_{j=0}^{n-1} \sum_{w \in f^{-j}\left(c^{\prime}\right)} \log \left[f^{n}(w), w\right]-\frac{2 d^{n}-2}{n d^{n}} \cdot 2 \sup _{\mathrm{P}^{1}}\left|g_{f}\right|,
\end{aligned}
$$

where the sums take into account the appropriate multiplicities of $c, c^{\prime}$, and $w$.

We can fix $L>1$ such that $f: \mathbb{P}^{1} \rightarrow \mathbb{P}^{1}$ is $L$-Lipschitz continuous with respect to the normalized chordal metric (for non-archimedean $K$, see, e.g., [22, Theorem 2.14]). Then for every $c^{\prime} \in C(f) \backslash S A T(f)$, every $j \in$ $\{0,1,2, \ldots, n-1\}$, and every $w \in f^{-j}\left(c^{\prime}\right), L^{n}\left[f^{n}(w), w\right] \geq L^{j}\left[f^{n}(w), w\right] \geq$ $\left[f^{j}\left(f^{n}(w)\right), f^{j}(w)\right]=\left[f^{n}\left(c^{\prime}\right), c^{\prime}\right]$, so that

$$
\log \left[f^{n}(w), w\right] \geq \log \left[f^{n}\left(c^{\prime}\right), c^{\prime}\right]-n \log L
$$

Recall the definition of the Berkovich Julia and Fatou sets $\mathrm{J}(f)$ and $\mathrm{F}(f)$ in Section 2. We claim that for every $c^{\prime} \in C(f) \backslash S A T(f)$,

$$
(0 \geq) \log \left[f^{n}\left(c^{\prime}\right), c^{\prime}\right] \geq\left\{\begin{array}{ll}
O(1) & \text { if } c^{\prime} \in \mathrm{F}(f) \\
O(n) & \text { if } c^{\prime} \in \mathrm{J}(f)
\end{array} \quad \text { as } n \rightarrow \infty ;\right.
$$

indeed, in the former case, if $\liminf _{n \rightarrow \infty}\left[f^{n}\left(c^{\prime}\right), c^{\prime}\right]=0$ for some $c^{\prime} \in(C(f) \cap$ $\mathrm{F}(f)) \backslash S A T(f)$, then the Berkovich Fatou component $U$ of $f$ containing $c^{\prime}$ is cyclic under $f$, i.e., $f^{p}(U)=U$ for some $p \in \mathbb{N}$. Since $c^{\prime} \in C(f) \cap U$, $f^{p}: U \rightarrow U$ is not injective, and by the classification of cyclic Berkovich Fatou components of $f$ (see Section 2), $U$ is an immediate attracting basin of an either (super)attracting or parabolic cycle of $f$ in $\mathbb{P}^{1}$. Then since $c^{\prime} \notin$ $S A T(f), \liminf _{n \rightarrow \infty}\left[f^{n}\left(c^{\prime}\right), c^{\prime}\right]>0$, which is a contradiction. On the other hand, in the latter case, by (the proof of) Przytycki's lemma [20, Lemma 1], it holds that for every $c^{\prime} \in C(f) \cap \mathrm{J}(f)$ and every $n \in \mathbb{N},\left[f^{n}\left(c^{\prime}\right), c^{\prime}\right] \geq$ $1 /\left(20 L^{n}\right)$. 
Hence the claim holds. Now we have

$$
\begin{aligned}
& \frac{1}{n d^{n}} \sum_{c^{\prime} \in C(f) \backslash S A T(f)} \sum_{j=0}^{n-1} \sum_{w \in f^{-j}\left(c^{\prime}\right)} \log \left[f^{n}(w), w\right] \\
& \geq \frac{1}{n d^{n}} \sum_{c^{\prime} \in C(f) \backslash S A T(f)} \sum_{j=0}^{n-1} d^{j}\left(\log \left[f^{n}\left(c^{\prime}\right), c^{\prime}\right]-n \log L\right) \\
& \quad \geq \frac{2 d-2}{n d^{n}} \cdot O(n) \cdot \sum_{j=0}^{n-1} d^{j}=O(1) \quad \text { as } n \rightarrow \infty,
\end{aligned}
$$

and the proof of Lemma 3.2 is complete.

The following technical and elementary lemma is useful.

Lemma 3.3. There exists $\delta>0$ such that for every $a \in S A T(f)$ and every $c \in C(f)$ satisfying $\bigcup_{j \in \mathbb{N} \cup\{0\}} f^{-j}(c) \neq\{a\}$,

$$
\inf \left\{[a, w]: w \in\left(\bigcup_{j \in \mathbb{N} \cup\{0\}} f^{-j}(c)\right) \backslash\{a\}\right\} \geq \delta .
$$

Moreover, if $a \in S A T(f)$ and $c \in C(f)$ satisfy $\bigcup_{j \in \mathbb{N} \cup\{0\}} f^{-j}(c)=\{a\}$, then $a \in \operatorname{Fix}(f)$. In particular, $\bigcup_{j \in \mathbb{N} \cup\{0\}} f^{-j}(c) \subset \operatorname{Fix}(f)$.

Proof. Let $a \in S A T(f)$. Then there is $p \in \mathbb{N}$ such that $f^{p}(a)=a$ and $f^{j}(a) \neq a$ for every $j \in\{1,2, \ldots, p-1\}$. We can fix an open neighborhood $U$ of $a$ in $\mathbb{P}^{1}$ so small that $f^{p}(U) \subset U$ by the Taylor expansion of $f^{p}$ at $a$ and that $f^{\ell}(U)(\ell \in\{0,1,2, \ldots, p-1\})$ are mutually disjoint. Set $\mathcal{O}_{a}:=$ $\left\{f^{\ell}(a): \ell \in\{0,1,2, \ldots, p-1\}\right\}$ and $\mathcal{U}:=\bigcup_{\ell=0}^{p-1} f^{\ell}(U)$, so that $f(\mathcal{U}) \subset \mathcal{U}$, $\mathcal{U} \cap U=U$ and $\mathcal{O}_{a} \cap U=\{a\}$. Decreasing $U$ if necessary, we can assume $f^{-1}\left(\mathcal{O}_{a}\right) \cap \mathcal{U} \subset \mathcal{O}_{a}$ and $\mathcal{U} \cap C(f) \subset \mathcal{O}_{a}$ by $\# f^{-1}\left(\mathcal{O}_{a}\right)<\infty$ and $\# C(f)<\infty$, respectively.

Let $c \in C(f)$ satisfy $\bigcup_{j \in \mathbb{N} \cup\{0\}} f^{-j}(c) \neq\{a\}$. If $\left(\bigcup_{j \in \mathbb{N} \cup\{0\}} f^{-j}(c)\right) \cap(U \backslash$ $\{a\}) \neq \emptyset$, then $c \in \mathcal{U} \cap C(f)$ by $f(\mathcal{U}) \subset \mathcal{U}$, and $c \in \mathcal{O}_{a}$ by $\mathcal{U} \cap C(f) \subset \mathcal{O}_{a}$. Hence by $f^{-1}\left(\mathcal{O}_{a}\right) \cap \mathcal{U} \subset \mathcal{O}_{a}$, we have $\left(\bigcup_{j \in \mathbb{N} \cup\{0\}} f^{-j}(c)\right) \cap \mathcal{U} \subset \mathcal{O}_{a}$, so $\left(\bigcup_{j \in \mathbb{N} \cup\{0\}} f^{-j}(c)\right) \cap U \subset \mathcal{O}_{a} \cap U=\{a\}$, which is a contradiction. Hence

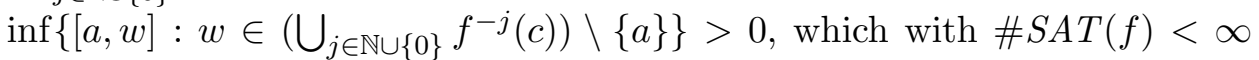
and $\# C(f)<\infty$ completes the proof of the former assertion. The latter assertion is obvious.

\section{Lemma 3.4.}

$$
\sup _{n \in \mathbb{N}}\left(\sup _{c \in\left(\bigcup_{j=0}^{n-1} f^{-j}(C(f) \cap S A T(f))\right) \backslash \operatorname{Fix}\left(f^{n}\right)}\left|\Phi_{g_{f}}\left(f^{n}(c), c\right)\right|\right) \leq-\log \delta+2 \sup _{\mathrm{P}^{1}}\left|g_{f}\right|<\infty .
$$

Proof. For every $n \in \mathbb{N}$ and every $c \in\left(\bigcup_{j=0}^{n-1} f^{-j}(C(f) \cap S A T(f))\right) \backslash \operatorname{Fix}\left(f^{n}\right)$, we have $f^{n}(c) \in S A T(f)$ and $c \in \bigcup_{j=0}^{n-1} f^{-j}\left(c^{\prime}\right)$ for some $c^{\prime} \in C(f)$. Since $c \notin$ $\operatorname{Fix}\left(f^{n}\right)$, by the latter assertion of Lemma 3.3, $\bigcup_{j \in \mathbb{N} \cup\{0\}} f^{-j}\left(c^{\prime}\right) \neq\left\{f^{n}(c)\right\}$. 
Hence by (3.8), we have either $c=f^{n}(c)$ or $\left[f^{n}(c), c\right] \geq \delta$, but the former possibility does not occur since $c \notin \operatorname{Fix}\left(f^{n}\right)$.

Hence $\inf _{n \in \mathbb{N}}\left(\inf _{c \in\left(\bigcup_{j=0}^{n-1} f^{-j}(C(f) \cap S A T(f))\right) \backslash \operatorname{Fix}\left(f^{n}\right)}\left[f^{n}(c), c\right]\right) \geq \delta$. Now the proof is complete by the definition of $\Phi_{g_{f}}$.

Lemma 3.5.

$$
\begin{aligned}
& \sup _{n \in \mathbb{N}}\left(\sup _{c \in C\left(f^{n}\right) \backslash \operatorname{Fix}\left(f^{n}\right)}\left|\int_{S A T(f)} \Phi_{g_{f}}(c, \cdot) \mathrm{d}\left[f^{n}=\mathrm{Id}\right](\cdot)\right|\right) \\
& \leq\left(-\log \delta+2 \sup _{\mathrm{P}^{1}}\left|g_{f}\right|\right)\left(\sup _{n \in \mathbb{N}}\left(\left[f^{n}=\mathrm{Id}\right](S A T(f))\right)\right)<\infty .
\end{aligned}
$$

Proof. For every $n \in \mathbb{N}$, every $c \in C\left(f^{n}\right) \backslash \operatorname{Fix}\left(f^{n}\right)$, and every $w \in S A T(f) \cap$ $\operatorname{Fix}\left(f^{n}\right)$, we have $c \in \bigcup_{j=0}^{n-1} f^{-j}\left(c^{\prime}\right)$ for some $c^{\prime} \in C(f)$. Since $c \notin \operatorname{Fix}\left(f^{n}\right)$, by the latter assertion of Lemma 3.3. $\bigcup_{j \in \mathbb{N} \cup\{0\}} f^{-j}\left(c^{\prime}\right) \neq\{w\}$. Hence by (3.8), we have either $c=w \in \operatorname{Fix}\left(f^{n}\right)$ or $[c, w] \geq \delta$, but the former possibility does not occur since $c \notin \operatorname{Fix}\left(f^{n}\right)$.

Hence $\inf _{n \in \mathbb{N}}\left(\inf _{c \in C\left(f^{n}\right) \backslash \operatorname{Fix}\left(f^{n}\right), w \in S A T(f) \cap \operatorname{Fix}\left(f^{n}\right)}[c, w]\right) \geq \delta$. Now the proof is complete by the definition of $\Phi_{g_{f}}$ and $\sup _{n \in \mathbb{N}}\left(\left[f^{n}=\mathrm{Id}\right](S A T(f))\right)<$ $\infty$.

\section{Lemma 3.6.}

$$
\begin{aligned}
& \sup _{n \in \mathbb{N}}\left(\sup _{c \in C\left(f^{n}\right) \cap \operatorname{Fix}\left(f^{n}\right)}\left|\int_{S A T(f) \backslash\{c\}} \Phi_{g_{f}}(c, \cdot) \mathrm{d}\left[f^{n}=\mathrm{Id}\right](\cdot)\right|\right) \\
\leq & \left(-\log \left(\inf _{c, c^{\prime} \in S A T(f): c \neq c^{\prime}}\left[c, c^{\prime}\right]\right)+2 \sup _{\mathrm{P}^{1}}\left|g_{f}\right|\right)\left(\sup _{n \in \mathbb{N}}\left(\left[f^{n}=\mathrm{Id}\right](S A T(f))\right)\right)<\infty .
\end{aligned}
$$

Proof. Since \#SAT $(f)<\infty$, we have $\inf _{c, c^{\prime} \in S A T(f): c \neq c^{\prime}}\left[c, c^{\prime}\right] \in(0,1)$.

Now the proof is complete by $\bigcup_{n \in \mathbb{N}}\left(C\left(f^{n}\right) \cap \operatorname{Fix}\left(f^{n}\right)\right)=S A T(f)$, the definition of $\Phi_{g_{f}}$, and $\sup _{n \in \mathbb{N}}\left(\left[f^{n}=\mathrm{Id}\right](S A T(f))\right)<\infty$.

By Lemmas 3.1, 3.2, 3.4, 3.5, 3.6, we have

$$
\begin{gathered}
\frac{1}{d^{n}} \int_{\mathrm{P}^{1} \backslash S A T(f)} \log \left(f^{\#}\right) \mathrm{d}\left[f^{n}=\mathrm{Id}\right]-\frac{\left[f^{n}=\mathrm{Id}\right]\left(\mathrm{P}^{1} \backslash S A T(f)\right)}{d^{n}} L(f) \\
=O(1)+3 \cdot \frac{2 d^{n}-2}{n d^{n}} \cdot O(1)-\frac{2-2 d^{-n}}{n} \int_{\mathrm{P}^{1}} \Phi\left(f^{n}, \mathrm{Id}\right)_{g_{f}} \mathrm{~d} \mu_{f} \\
=-\frac{2-2 d^{-n}}{n} \int_{\mathrm{P}^{1}} \Phi\left(f^{n}, \mathrm{Id}\right)_{g_{f}} \mathrm{~d} \mu_{f}+O(1) \quad \text { as } n \rightarrow \infty .
\end{gathered}
$$

By an argument similar to (and simpler than) the above, the following also holds.

\section{Lemma 3.7.}

$$
\begin{gathered}
\frac{1}{d^{n}} \int_{\mathrm{P}^{1} \backslash S A T(f)} \log \left(f^{\#}\right) \mathrm{d}\left[f^{n}=\mathrm{Id}\right]-\frac{\left[f^{n}=\mathrm{Id}\right]\left(\mathrm{P}^{1} \backslash S A T(f)\right)}{d^{n}} L(f) \\
=-\frac{2 d-2}{d^{n}} \int_{\mathrm{P}^{1}} \Phi\left(f^{n}, \mathrm{Id}\right)_{g_{f}} \mathrm{~d} \mu_{f}+O\left(n d^{-n}\right) \quad \text { as } n \rightarrow \infty
\end{gathered}
$$


Proof. In the following, the sums over subsets of $C(f)$ take into account the multiplicity of each $c$ as a critical point of $f$.

For every $n \in \mathbb{N}$, integrating both sides in (2.6) itself against $\mathrm{d}\left[f^{n}=\mathrm{Id}\right]$ over $\mathbb{P}^{1} \backslash S A T(f)$, since $f_{*}\left[f^{n}=\mathrm{Id}\right]=\left[f^{n}=\mathrm{Id}\right]$ on $\mathrm{P}^{1} \backslash S A T(f)$, we have

$$
\begin{aligned}
\int_{\mathrm{P}^{1} \backslash S A T(f)} \log \left(f^{\#}\right) \mathrm{d}\left[f^{n}=\mathrm{Id}\right]-\left(\left[f^{n}=\right.\right. & \left.\mathrm{Id}]\left(\mathrm{P}^{1} \backslash S A T(f)\right)\right) \cdot L(f) \\
& =\sum_{c \in C(f)} U_{g_{f},\left[f^{n}=\mathrm{Id}\right] \mid\left(\mathrm{P}^{1} \backslash S A T(f)\right)}(c),
\end{aligned}
$$

and by (3.6) applied to $f^{n}$, for each $c \in C(f)\left(\subset C\left(f^{n}\right)\right)$,

$$
\begin{aligned}
\left.U_{g_{f},\left[f^{n}\right.}=\mathrm{Id}\right] \mid\left(\mathrm{P}^{1} \backslash S A T(f)\right) & (c)+\int_{\mathrm{P}^{1}} \Phi\left(f^{n}, \mathrm{Id}\right)_{g_{f}} \mathrm{~d} \mu_{f} \\
& =\left\{\begin{array}{cl}
\Phi_{g_{f}}\left(f^{n}(c), c\right)-\int_{S A T(f)} \Phi_{g_{f}}(c, \cdot) \mathrm{d}\left[f^{n}=\mathrm{Id}\right](\cdot) & \text { if } c \notin \operatorname{Fix}\left(f^{n}\right), \\
-\int_{S A T(f) \backslash\{c\}} \Phi_{g_{f}}(c, \cdot) \mathrm{d}\left[f^{n}=\mathrm{Id}\right](\cdot) & \text { if } c \in \operatorname{Fix}\left(f^{n}\right),
\end{array}\right.
\end{aligned}
$$

where $g_{f^{n}}=g_{f}, \mu_{f^{n}}=\mu_{f}$, and $S A T\left(f^{n}\right)=S A T(f) \cap \operatorname{Fix}\left(f^{n}\right)$. Hence for every $n \in \mathbb{N}$,

$$
\begin{aligned}
& \sum_{c \in C(f)} U_{g_{f},\left[f^{n}=\mathrm{Id}\right] \mid\left(\mathrm{P}^{1} \backslash S A T(f)\right)}(c)+(2 d-2) \int_{\mathrm{P}^{1}} \Phi\left(f^{n}, \mathrm{Id}\right)_{g_{f}} \mathrm{~d} \mu_{f} \\
= & \sum_{c \in C(f) \backslash \operatorname{Fix}\left(f^{n}\right)} \Phi_{g_{f}}\left(f^{n}(c), c\right)-\sum_{c \in C(f) \backslash \operatorname{Fix}\left(f^{n}\right)} \int_{S A T(f)} \Phi_{g_{f}}(c, \cdot) \mathrm{d}\left[f^{n}=\mathrm{Id}\right](\cdot) \\
- & \sum_{c \in C(f) \cap \operatorname{Fix}\left(f^{n}\right)} \int_{S A T(f) \backslash\{c\}} \Phi_{g_{f}}(c, \cdot) \mathrm{d}\left[f^{n}=\mathrm{Id}\right](\cdot)
\end{aligned}
$$

so, by $(C(f) \backslash S A T(f)) \backslash \operatorname{Fix}\left(f^{n}\right)=C(f) \backslash S A T(f)$, we have

$$
\begin{aligned}
& \frac{1}{d^{n}} \int_{\mathrm{P}^{1} \backslash S A T(f)} \log \left(f^{\#}\right) \mathrm{d}\left[f^{n}=\mathrm{Id}\right]-\frac{\left[f^{n}=\mathrm{Id}\right]\left(\mathrm{P}^{1} \backslash S A T(f)\right)}{d^{n}} L(f) \\
\text { (3.1]) } \quad= & \frac{1}{d^{n}} \sum_{c \in C(f) \backslash S A T(f)} \Phi_{g_{f}}\left(f^{n}(c), c\right) \\
& +\frac{1}{d^{n}} \sum_{c \in(C(f) \cap S A T(f)) \backslash \operatorname{Fix}\left(f^{n}\right)} \Phi_{g_{f}}\left(f^{n}(c), c\right) \\
& -\frac{1}{d^{n}} \sum_{c \in C(f) \backslash \operatorname{Fix}\left(f^{n}\right)} \int_{S A T(f)} \Phi_{g_{f}}(c, \cdot) \mathrm{d}\left[f^{n}=\mathrm{Id}\right](\cdot) \\
& -\frac{1}{d^{n}} \sum_{c \in C(f) \cap \operatorname{Fix}\left(f^{n}\right)} \int_{S A T(f) \backslash\{c\}} \Phi_{g_{f}}(c, \cdot) \mathrm{d}\left[f^{n}=\mathrm{Id}\right](\cdot) \\
& -\frac{2 d-2}{d^{n}} \int_{\mathrm{P}^{1}} \Phi\left(f^{n}, \mathrm{Id}\right)_{g_{f}} \mathrm{~d} \mu_{f} .
\end{aligned}
$$


By (3.7) and the definition of $\Phi_{g_{f}}$, the term (3.11) is estimated as

$$
\begin{aligned}
\frac{2 d-2}{d^{n}} \cdot 2 \sup _{\mathrm{P}^{1}}\left|g_{f}\right| & \geq \frac{1}{d^{n}} \sum_{c \in C(f) \backslash S A T(f)} \Phi_{g_{f}}\left(f^{n}(c), c\right) \\
& \geq \frac{1}{d^{n}} \sum_{c \in C(f) \backslash S A T(f)} \log \left[f^{n}(c), c\right]-\frac{2 d-2}{d^{n}} \cdot 2 \sup _{\mathrm{P}^{1}}\left|g_{f}\right| \\
& \geq \frac{2 d-2}{d^{n}} \cdot O(n)+O\left(d^{-n}\right)=O\left(n d^{-n}\right) \quad \text { as } n \rightarrow \infty,
\end{aligned}
$$

and since $C(f) \subset C\left(f^{n}\right)$, by Lemmas 3.4, 3.5, and 3.6 (or by arguments similar to and simpler than those in their proofs), the terms (3.2]), (3.3il), and (3.4I) have the order $\left((2 d-2) / d^{n}\right) \cdot O(1)=O\left(d^{-n}\right)$ as $n \rightarrow \infty$.

Now the proof is complete.

By (3.9) and (3.10), we have

$$
\begin{aligned}
-\frac{2-2 d^{-n}}{n} \int_{\mathrm{P}^{1}} & \Phi\left(f^{n}, \mathrm{Id}\right)_{g_{f}} \mathrm{~d} \mu_{f}+O(1) \\
& =-\frac{2 d-2}{d^{n}} \int_{\mathrm{P}^{1}} \Phi\left(f^{n}, \mathrm{Id}\right)_{g_{f}} \mathrm{~d} \mu_{f}+O\left(n d^{-n}\right) \quad \text { as } n \rightarrow \infty,
\end{aligned}
$$

which with $(0 \neq)-\left(2-2 d^{-n}\right) / n+(2 d-2) / d^{n}=O\left(n^{-1}\right)$ as $n \rightarrow \infty$ yields

$$
\int_{\mathrm{P}^{1}} \Phi\left(f^{n}, \mathrm{Id}\right)_{g_{f}} \mathrm{~d} \mu_{f}=n \cdot O(1)=O(n) \quad \text { as } n \rightarrow \infty .
$$

Proof of Theorem 1. Once (3.10) and (3.11) are at our disposal, we have

$$
\begin{aligned}
\frac{1}{d^{n}} \int_{\mathrm{P}^{1} \backslash S A T(f)} \log \left(f^{\#}\right) \mathrm{d}\left[f^{n}=\mathrm{Id}\right]-\frac{\left[f^{n}=\mathrm{Id}\right]\left(\mathrm{P}^{1} \backslash S A T(f)\right)}{d^{n}} L(f) & \\
=O\left(n d^{-n}\right) & \text { as } n \rightarrow \infty,
\end{aligned}
$$

the left hand side of which is computed as

$$
\begin{aligned}
& \frac{1}{d^{n}} \int_{\mathrm{P}^{1} \backslash S A T(f)} \log \left(f^{\#}\right) \mathrm{d}\left[f^{n}=\mathrm{Id}\right]-\frac{\left[f^{n}=\mathrm{Id}\right]\left(\mathrm{P}^{1} \backslash S A T(f)\right)}{d^{n}} L(f) \\
& \quad=\frac{1}{n d^{n}} \sum_{w \in \operatorname{Fix}\left(f^{n}\right) \backslash S A T(f)} \log \left|\left(f^{n}\right)^{\prime}(w)\right|-L(f)+O\left(d^{-n}\right) \quad \text { as } n \rightarrow \infty
\end{aligned}
$$

by the chain rule, $\left[f^{n}=\mathrm{Id}\right]\left(\mathrm{P}^{1}\right)=d^{n}+1$, and $\sup _{n \in \mathbb{N}}\left(\left[f^{n}=\mathrm{Id}\right](S A T(f))\right)<$ $\infty$. Now the proof of (1.2) is complete.

Let us show (1.3) using (1.2): for the details on the Möbius function $\mu: \mathbb{N} \rightarrow\{-1,0,1\}$, which satisfies $\mu(1)=1$ by the definition, and the Möbius inversion formula below, see, e.g, [1, §2].

For every $n \in \mathbb{N}$, using the chain rule, we have

$\frac{1}{n} \sum_{w \in \operatorname{Fix}\left(f^{n}\right) \backslash S A T(f)} \log \left|\left(f^{n}\right)^{\prime}(w)\right|=\sum_{m \in \mathbb{N}: m \mid n} \frac{1}{m} \sum_{w \in \mathrm{Fix}^{*}\left(f^{m}\right) \backslash S A T(f)} \log \left|\left(f^{m}\right)^{\prime}(w)\right|$, 
which is equivalent to

$$
\begin{aligned}
& \frac{1}{n} \sum_{w \in \mathrm{Fix}^{*}\left(f^{n}\right) \backslash S A T(f)} \log \left|\left(f^{n}\right)^{\prime}(w)\right| \\
& =\sum_{m \in \mathbb{N}: m \mid n} \mu\left(\frac{n}{m}\right) \cdot \frac{1}{m} \sum_{w \in \operatorname{Fix}\left(f^{m}\right) \backslash S A T(f)} \log \left|\left(f^{m}\right)^{\prime}(w)\right|
\end{aligned}
$$

by the Möbius inversion formula.

By \#SAT $(f)<\infty$, for every $n \in \mathbb{N}$ large enough, we have $\operatorname{Fix}^{*}\left(f^{n}\right) \backslash$ $S A T(f)=\operatorname{Fix}^{*}\left(f^{n}\right)$. Hence by (3.12), we have

$$
\begin{gathered}
\left|\frac{1}{n d^{n}} \sum_{w \in \mathrm{Fix}^{*}\left(f^{n}\right)} \log \right|\left(f^{n}\right)^{\prime}(w)\left|-\frac{1}{n d^{n}} \sum_{w \in \operatorname{Fix}\left(f^{n}\right) \backslash S A T(f)} \log \right|\left(f^{n}\right)^{\prime}(w)|| \\
\leq \frac{1}{d^{n}} \sum_{m \in \mathbb{N}: m \mid n \text { and } m<n} d^{m}\left(\frac{1}{m d^{m}} \sum_{w \in \operatorname{Fix}\left(f^{m}\right) \backslash S A T(f)} \log \left|\left(f^{m}\right)^{\prime}(w)\right|\right) \\
\leq O(1) \cdot \frac{1}{d^{n}} \sum_{m=1}^{n / 2} d^{m}=O\left(d^{-n / 2}\right) \quad \text { as } n \rightarrow \infty,
\end{gathered}
$$

where the second inequality is by (1.2) and $\sup \{m \in \mathbb{N}: m \mid n$ and $m<n\} \leq$ $n / 2$. Now the proof of (1.3) is complete by (1.2).

Remark 3.8. The order estimate

$$
\frac{1}{m d^{m}} \sum_{w \in \operatorname{Fix}\left(f^{m}\right) \backslash S A T(f)} \log \left|\left(f^{m}\right)^{\prime}(w)\right|=O(1) \quad \text { as } m \rightarrow \infty
$$

is immediate if $f$ has at most finitely many attracting periodic points in $\mathbb{P}^{1}$.

\section{Proof of Theorem 2}

Suppose in addition that $f$ has at most finitely many attracting periodic points in $\mathbb{P}^{1}$, or equivalently, that there exists $N \in \mathbb{N}$ such that for every $n \geq N$ and every $w \in \operatorname{Fix}^{*}\left(f^{n}\right)$, we have $\left|\left(f^{n}\right)^{\prime}(w)\right| \geq 1$. Hence (1.3) implies (1.3il).

For every $n \geq N$,

$$
\begin{aligned}
& \frac{1}{n d^{n}} \sum_{w \in \operatorname{Fix}\left(f^{n}\right) \backslash S A T(f)} \log \left|\left(f^{n}\right)^{\prime}(w)\right| \\
= & \frac{1}{n d^{n}} \sum_{m \in \mathbb{N}: m \mid n \text { and } m \geq N}\left(\sum_{w \in R_{m}^{*}(f)} \log \left|\left(f^{n}\right)^{\prime}(w)\right|\right)+O\left(\left(n d^{n}\right)^{-1}\right) \\
= & \frac{1}{n d^{n}} \sum_{m \in \mathbb{N}: m \mid n}\left(\sum_{w \in R_{m}^{*}(f)} \log \left|\left(f^{n}\right)^{\prime}(w)\right|\right)+O\left(\left(n d^{n}\right)^{-1}\right) \\
= & \frac{1}{n d^{n}} \sum_{w \in R\left(f^{n}\right)} \log \left|\left(f^{n}\right)^{\prime}(w)\right|+O\left(\left(n d^{n}\right)^{-1}\right) \quad \text { as } n \rightarrow \infty,
\end{aligned}
$$

so (1.2) implies (1.21). 
Acknowledgement. The author thanks the referee for a very careful scrutiny and invaluable comments. This research was partially supported by JSPS Grant-in-Aid for Young Scientists (B), 24740087.

\section{REFERENCES}

[1] Apostol, T. M. Introduction to analytic number theory, Springer (1976).

[2] BAKER, M. A finiteness theorem for canonical heights attached to rational maps over function fields, Journal für die reine und angewandte Mathematik (Crelles Journal), 626 (2009), 205-233.

[3] BAKer, M. H. and Rumely, R. Equidistribution of small points, rational dynamics, and potential theory, Ann. Inst. Fourier (Grenoble), 56, 3 (2006), 625-688.

[4] Baker, M. and Rumely, R. Potential theory and dynamics on the Berkovich projective line, Vol. 159 of Mathematical Surveys and Monographs, American Mathematical Society, Providence, RI (2010).

[5] Benedetto, R. Non-archimedean dynamics in dimension one: lecture notes, Preprint. Available at http://math.arizona.edu/ ${ }^{\sim}$ swc/aws/2010/ (2010).

[6] Berteloot, F. Lyapunov exponent of a rational map and multipliers of repelling cycles, Riv. Mat. Univ. Parma (N.S.), 1, 2 (2010), 263-269.

[7] Berteloot, F., Dupont, C. and Molino, L. Normalization of bundle holomorphic contractions and applications to dynamics, Ann. Inst. Fourier (Grenoble), 58, 6 (2008), 2137-2168.

[8] Berteloot, F. and Mayer, V. Rudiments de dynamique holomorphe, Vol. 7 of Cours Spécialisés [Specialized Courses], Société Mathématique de France, Paris (2001).

[9] Chambert-Loir, A. Mesures et équidistribution sur les espaces de Berkovich, $J$. Reine Angew. Math., 595 (2006), 215-235.

[10] DeMarco, L. Dynamics of rational maps: Lyapunov exponents, bifurcations, and capacity, Math. Ann., 326, 1 (2003), 43-73.

[11] Favre, C. and Jonsson, M. The valuative tree, Vol. 1853 of Lecture Notes in Mathematics, Springer-Verlag, Berlin (2004).

[12] Favre, C. and Rivera-Letelier, J. Théorie ergodique des fractions rationnelles sur un corps ultramétrique, Proc. Lond. Math. Soc. (3), 100, 1 (2010), 116-154.

[13] Jonsson, M. Sums of Lyapunov exponents for some polynomial maps of $\mathbf{C}^{2}$, Ergodic Theory Dynam. Systems, 18, 3 (1998), 613-630.

[14] Jonsson, M. Dynamics on Berkovich spaces in low dimensions, ArXiv e-prints (Jan. 2012).

[15] LJUBich, M. J. Entropy properties of rational endomorphisms of the Riemann sphere, Ergodic Theory Dynam. Systems, 3, 3 (1983), 351-385.

[16] Milnor, J. Dynamics in one complex variable, Vol. 160 of Annals of Mathematics Studies, Princeton University Press, Princeton, NJ, third edition (2006).

[17] Okuyama, Y. Repelling periodic points and logarithmic equidistribution in nonarchimedean dynamics., Acta Arith., 152, 3 (2012), 267-277.

[18] OKuyama, Y. Adelic equidistribution, characterization of equidistribution, and a general equidistribution theorem in non-archimedean dynamics, Acta. Arith., 161, 2 (2013), 101-125.

[19] OKuyama, Y. Fekete configuration, quantitative equidistribution and wandering critical orbits in non-archimedean dynamics, Math. Z., 273, 3-4 (2013), 811-837.

[20] Przytycki, F. Lyapunov characteristic exponents are nonnegative, Proc. Amer. Math. Soc., 119, 1 (1993), 309-317.

[21] Rivera-Letelier, J. Dynamique des fonctions rationnelles sur des corps locaux, Astérisque, 287 (2003), xv, 147-230, Geometric methods in dynamics. II.

[22] Silverman, J. H. The arithmetic of dynamical systems, Vol. 241 of Graduate Texts in Mathematics, Springer, New York (2007).

[23] Sodin, M. Value distribution of sequences of rational functions, Entire and subharmonic functions, Vol. 11 of Adv. Soviet Math., Amer. Math. Soc., Providence, RI (1992), 7-20. 
[24] Szpiro, L. and Tucker, T. J. Equidistribution and generalized Mahler measures, Number Theory, Analysis and Geometry: In Memory of Serge Lang, Springer (2012), 609-638 (preprint version is available at http://arxiv.org/abs/math/0510404).

[25] Thuillier, A. Théorie du potentiel sur les courbes en géométrie analytique non archimédienne. Applications à la théorie d'Arakelov, $\mathrm{PhD}$ thesis, Université Rennes 1 (2005).

[26] Tsuji, M. Potential theory in modern function theory, Chelsea Publishing Co., New York (1975), Reprinting of the 1959 original.

Division of Mathematics, Kyoto Institute of Technology, Sakyo-Ku, Kyoto 606-8585 JAPAN.

E-mail address: okuyama@it.ac.jp 